OPEN

SUBJECT AREAS:

ECOLOGY

MOLECULAR BIOLOGY

ECOPHYSIOLOGY

Received

29 October 2013

Accepted

7 January 2014

Published

3 February 2014

Correspondence and requests for materials should be addressed to C.C.M. (ccm15@psu.

edu)

\section{Temperature alters Plasmodium blocking by Wolbachia}

\author{
Courtney C. Murdock, Simon Blanford, Grant L. Hughes, Jason L. Rasgon \& Matthew B. Thomas
}

Center for Infectious Disease Dynamics and Department of Entomology, Pennsylvania State University, University Park, PA, USA.

Very recently, the Asian malaria vector (Anopheles stephensi) was stably transinfected with the wAlbB strain of Wolbachia, inducing refractoriness to the human malaria parasite Plasmodium falciparum. However, conditions in the field can differ substantially from those in the laboratory. We use the rodent malaria $P$. yoelii, and somatically transinfected An. stephensi as a model system to investigate whether the transmission blocking potential of $w \mathrm{AlbB}$ is likely to be robust across different thermal environments. wAlbB reduced malaria parasite prevalence and oocyst intensity at $28^{\circ} \mathrm{C}$. At $24^{\circ} \mathrm{C}$ there was no effect on prevalence but a marked increase in oocyst intensity. At $20^{\circ} \mathrm{C}, w \mathrm{AlbB}$ had no effect on prevalence or intensity. Additionally, we identified a novel effect of wAlbB that resulted in reduced sporozoite development across temperatures, counterbalancing the oocyst enhancement at $24^{\circ} \mathrm{C}$. Our results demonstrate complex effects of temperature on the Wolbachia-malaria interaction, and suggest the impacts of transinfection might vary across diverse environments.

D espite intensive control efforts, malaria remains one of the most important infectious diseases worldwide ${ }^{1}$. Manipulation of mosquitoes to render them ineffective at transmitting parasites and pathogens could create exciting new opportunities for control of vector-borne diseases e.g. ${ }^{2-6}$. One of the most promising approaches involves transinfection of mosquitoes with strains of the maternally inherited endosymbiotic bacteria, Wolbachia (reviewed $\mathrm{in}^{7-12}$ ). Studies with a range of mosquito and parasite/pathogen species have shown Wolbachia to reduce vector competence and/or vectorial capacity ${ }^{4,13-26}$. Further, Wolbachia possesses a genetic drive mechanism (cytoplasmic incompatibility) that enhances its own spread through host populations by skewing normal Mendelian inheritance ratios ${ }^{27}$. Research on the use of Wolbachia for control of dengue has progressed to the level of controlled field releases of transinfected Aedes aegypti mosquitoes ${ }^{8,28}$. Research on malaria mosquito vectors has proven more challenging, but a stably transinfected line of Anopheles stephensi has recently been developed and evaluated in the laboratory ${ }^{4}$.

The mechanisms underlying the transmission blocking properties of Wolbachia remain unclear, but appear linked to aspects of mosquito immune function ${ }^{4,15,19-21,29-31}$, and possible competition for resources within the mosquito ${ }^{15}$. Recent research has demonstrated that mosquito immune function can vary considerably with modest changes in temperature ${ }^{32-34}$. It is also well established that growth of malaria parasites within the mosquito is strongly temperature-dependent ${ }^{35}$. Furthermore, studies from a wide diversity of non-vector systems illustrate that Wolbachia replication, dissemination, vertical transmission, fitness effects and the extent of cytoplasmic incompatibility can all vary with temperature (SI Table 1). This range of thermal sensitivities raises the possibility that patterns of Wolbachia-induced transmission blocking might depend strongly on the local environment. If so, insights gained under standard insectary conditions at $27^{\circ} \mathrm{C}$ may reveal little about the natural environments for malaria transmission where mean temperatures can range from 18 to $34^{\circ} \mathrm{C}$, and daily temperature variation frequently exceeds $10^{\circ} \mathrm{C}^{36-38}$. For example, blocking could be enhanced under certain conditions, increasing the effectiveness of the approach. Alternatively, variation in temperature might reduce blocking. The worst-case scenario is that malaria infection might actually be enhanced in certain environments by the interaction between Wolbachia and Plasmodium ${ }^{39}$.

As proof of principle, we use a rodent malaria P. yoelii and somatically transinfected An. stephensi as a model to investigate how changes in temperature influence the Wolbachia-malaria parasite interaction. If temperature significantly influences pathogen blocking, such effects would suggest that the impacts of transinfection cannot be determined from studies conducted under one set of conditions alone.

\section{Results}

Effects of temperature on Wolbachia density and mosquito survival. The density of Wolbachia increased over time (Fig. 1, Table 1), with mosquitoes sampled on day 20 exhibiting significantly higher densities of $w$ AlbB than 
Table 1 | Generalized linear model analysis of the effect of experimental treatment on wAlbB density, oocyst prevalence, and oocyst intensity

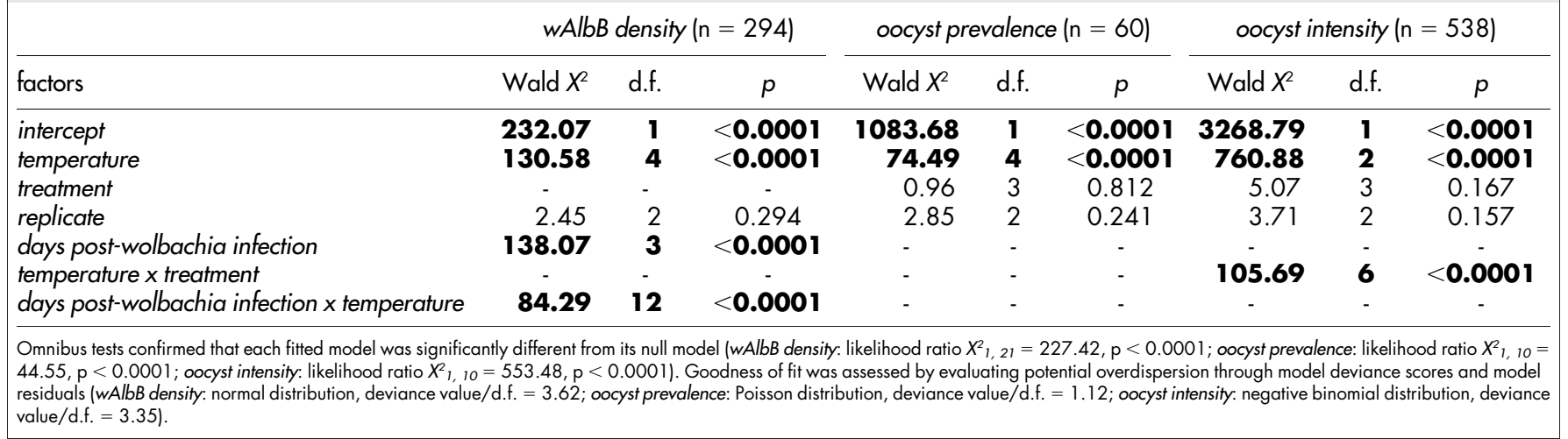

on days 16 ( $\mathrm{p}<0.0001), 12(\mathrm{p}<0.0001)$, and 8 ( $\mathrm{p}<0.0001)$ postinjection. Wolbachia densities were also significantly higher in the warmer temperatures of $26^{\circ} \mathrm{C}$ and $28^{\circ} \mathrm{C}$, compared with cooler temperatures $\left(20^{\circ} \mathrm{C}\right.$ vs. warmer temperatures, $\mathrm{p}<0.0001 ; 22^{\circ} \mathrm{C}$ vs. $24^{\circ} \mathrm{C}, \mathrm{p}=0.017 ; 22^{\circ} \mathrm{C}$ vs. $26^{\circ} \mathrm{C}$ and $28^{\circ} \mathrm{C}, \mathrm{p}<0.0001 ; 24^{\circ} \mathrm{C}$ vs. warmer temperatures, $\mathrm{p}<0.0001$; Table 1). A significant interaction between sampling day and temperature (days postWolbachia infection $x$ temperature) indicated that the rates of $w$ AlbB replication increased with warming temperatures, with no significant increases in $w$ AlbB densities occurring at $20^{\circ} \mathrm{C}$ and $22^{\circ} \mathrm{C}$ (Fig. 1).

There was no significant effect of temperature or Wolbachia infection on mosquito survival (Supplementary Information Text S2). There was a minor transient effect of micro-injection on mortality within the first 24-48 hrs, and this was consistent between the $w$ AlbB and Sua5B control treatments (Supplementary Information Text S2 Fig. 1; Sua5B cell control: $\left.X^{2}=106.28, \mathrm{p}<0.0001\right)$; $w$ AlbB: $X^{2}=$ $113.48, \mathrm{p}<0.0001)$. After the first two days of the experiment, the Sua5B control and $w$ AlbB survival curves resemble that of the unmanipulated population (Supplementary Information Text S2).

Effects of temperature on Plasmodium blocking. There was an effect of temperature on the probability of a mosquito becoming infected with $P$. yoelii (Table 1, Fig. 2a), with oocyst prevalence being significantly lower at $28^{\circ} \mathrm{C}(\mathrm{p}<0.0001)$ compared with cooler temperatures. There was no additional effect of Wolbachia on parasite prevalence. However, temperature and Wolbachia did interact to impact oocyst intensity (Table 1, Fig. 2b). At $20^{\circ} \mathrm{C}$, the number of oocysts per mosquito midgut did not differ between treatments. At $24^{\circ} \mathrm{C}$, infection with $w$ AlbB significantly facilitated the establishment of oocysts, such that the transinfected mosquitoes had more than double the oocyst burdens of the Sua5B control and unmanipulated control mosquitoes (Sua5B lysate, $\mathrm{p}<0.0001$; unmanipulated, $\mathrm{p}=0.01$ ). At $28^{\circ} \mathrm{C}$ on the other hand, infection with $w$ AlbB limited the number of establishing oocysts, reducing oocyst burdens by approximately $80 \%$ relative to control groups (Sua5B lysate, $\mathrm{p}<0.0001$; unmanipulated, $\mathrm{p}<0.0001$ ).

We next examined the number of sporozoites per oocyst to provide a measure of parasite replication rate. Infection with $w$ AlbB significantly reduced the number of sporozoites produced per oocyst across all temperatures, irrespective of Wolbachia infection status (Table 2, Fig. 2c). In general there was a relationship between the number of oocysts per midgut (oocyst intensity) and the number of sporozoites produced per oocyst. This negative effect of oocyst density was most marked in the transinfected mosquitoes and temperatures sub optimal for parasite development $\left(20^{\circ} \mathrm{C}\right.$ and $\left.28^{\circ} \mathrm{C}\right)$. This

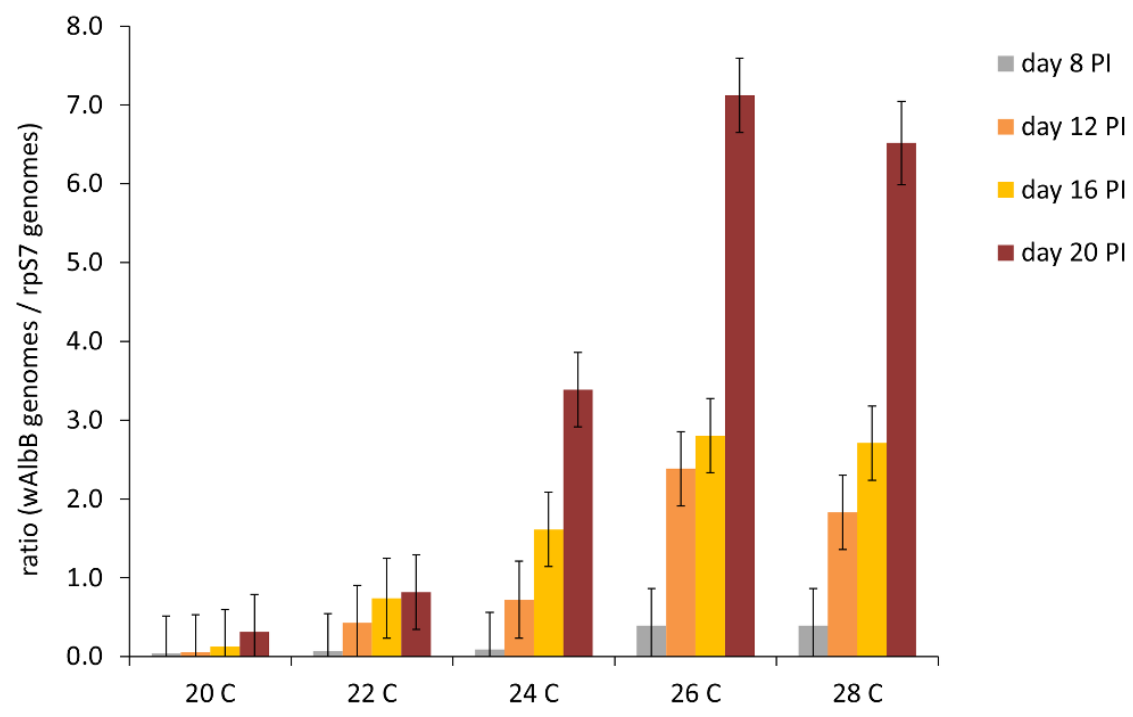

Figure $1 \mid$ Temperature, sampling time point, and their interaction significantly influenced wAlbB replication in whole mosquito carcasses. $w$ AlbB density (ratio of $w \mathrm{AlbB}$ to host $r p S 7$ genomes) is clearly mediated by temperature, with the rate of replication significantly increasing in mosquitoes housed at $26^{\circ} \mathrm{C}$ compared to those housed at $24^{\circ} \mathrm{C}$, and no significant changes through time in $w$ AlbB densities occurring in mosquitoes housed at $20^{\circ} \mathrm{C}$ and $22^{\circ} \mathrm{C}$. Bars around mean values represent standard errors. 
$\mathbf{a}$

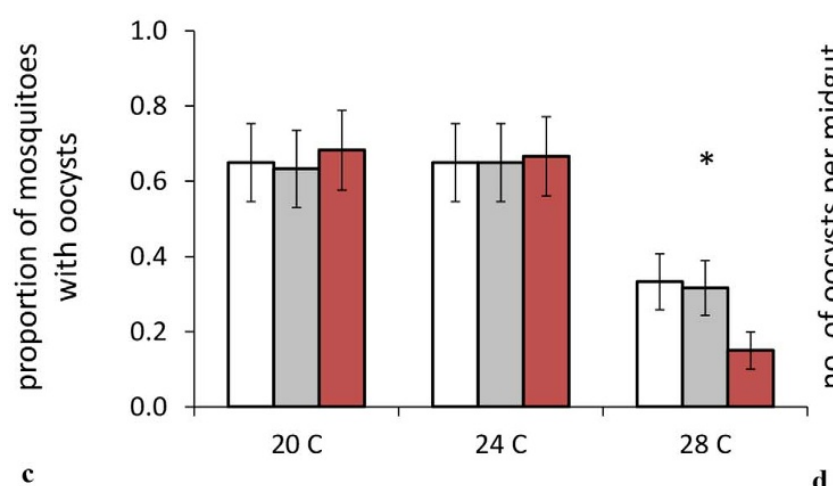

b

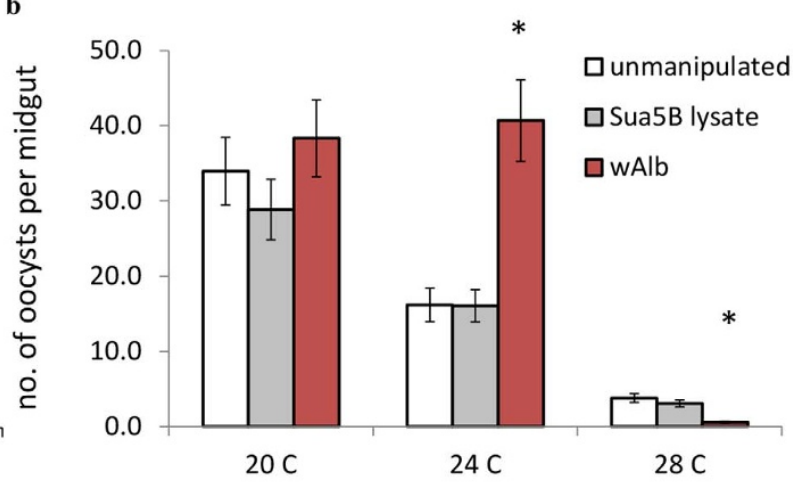

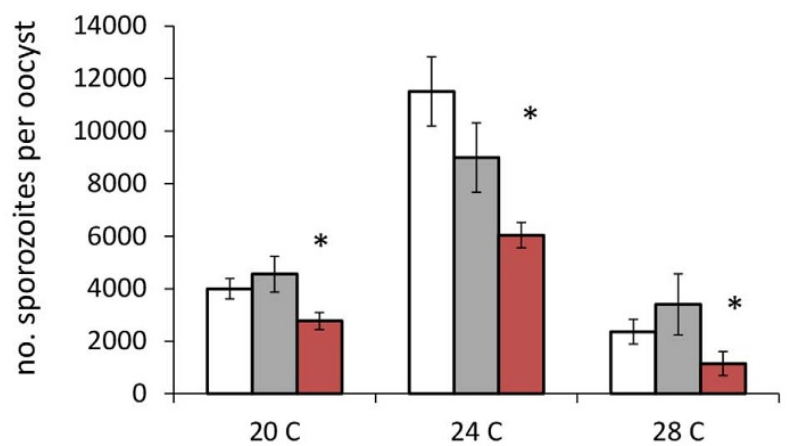

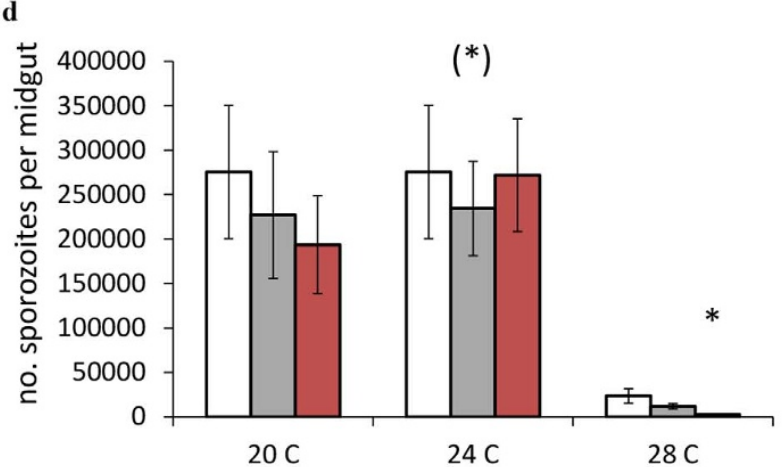

Figure $2 \mid$ Temperature shaped the wAlbB-malaria interaction in complex ways. (a) Temperature alone significantly affected oocyst prevalence (the proportion of mosquitoes with oocysts on their midguts), with significant declines in oocyst prevalence occurring at $28^{\circ} \mathrm{C}$ for all treatment groups (asterisk represents significant pairwise comparisons for each treatment group at $28^{\circ} \mathrm{C}$ with treatment groups placed at lower temperatures, $\mathrm{p}<0.05$ ). (b) Temperature significantly mediated the effect of $w$ AlbB on oocyst intensity (the number of oocysts per midgut). wAlbB infection either enhanced $\left(24^{\circ} \mathrm{C}\right)$, blocked $\left(28^{\circ} \mathrm{C}\right)$, or had no effect on the number of establishing oocysts $\left(20^{\circ} \mathrm{C}\right)$. (c) Infection with $w$ AlbB significantly reduced the number of sporozoites produced per oocyst across all temperature treatments. (d) Temperature significantly mediated the effect to $w \mathrm{AlbB}$ on the total number of sporozoites produced per mosquito, with significant declines in overall sporozoite production at $28^{\circ} \mathrm{C}$ in $w$ AlbB infected mosquitoes. Bars represent the unadjusted means of each response variable, while whiskers portray the standard error around the mean. Asterisks in (b-d) denote significant pair-wise comparisons within a temperature between $w \mathrm{AlbB}$ and the other treatment groups. The asterisk within parentheses at $24^{\circ} \mathrm{C}$ indicates a significant effect of $w \mathrm{AlbB}$ infection in explaining variation between the estimated marginal means in the full statistical model, but there is no significant effect when the unadjusted treatment means are compared.

was especially the case for $w$ AlbB infected mosquitoes at $28^{\circ} \mathrm{C}$ (Regression on model residuals: $B=-0.203, \mathrm{R}^{2}=0.867, \mathrm{~F}_{1,8}=$ $21.111, \mathrm{p}=0.002$ ), as illustrated by a significant interaction between treatment and oocyst intensity observed at $28^{\circ} \mathrm{C}$ (Table 2 ).
Finally, we used total sporozoites per mosquito midgut as a measure of overall infection intensity (and hence ultimate transmission potential). With this overall measure, GZLM model analyses predicted that infection with $w$ AlbB significantly reduced the total

Table 2 | Generalized linear model analysis of the effect of treatment on the number of sporozoites produced per oocyst and midgut analyzed independently for each experimental temperature

\begin{tabular}{|c|c|c|c|c|c|c|c|c|c|}
\hline \multirow[b]{2}{*}{ factors } & \multicolumn{3}{|c|}{$20^{\circ} \mathrm{C}(\mathrm{n}=116)$} & \multicolumn{3}{|c|}{$24^{\circ} \mathrm{C}(\mathrm{n}=118)$} & \multicolumn{3}{|c|}{$28^{\circ} \mathrm{C}(\mathrm{n}=42)$} \\
\hline & Wald $X^{2}$ & d.f. & $p$ & Wald $X^{2}$ & d.f. & $p$ & Wald $X^{2}$ & d.f. & $p$ \\
\hline \multicolumn{10}{|l|}{ sporozoites/oocyst } \\
\hline \multicolumn{10}{|c|}{ 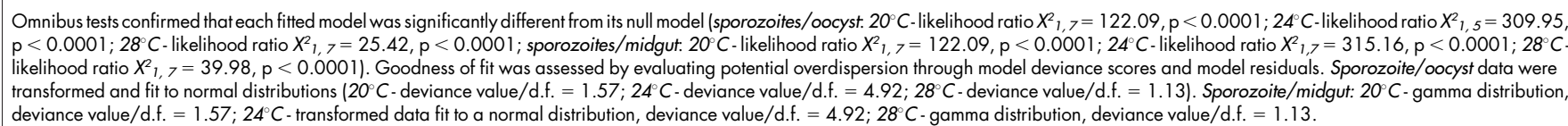 } \\
\hline
\end{tabular}


number of sporozoites produced at $24^{\circ} \mathrm{C}$ (unmanipulated vs. $w \mathrm{AlbB}$, $\mathrm{p}<0.0001$; Sua5B lysate vs. $w \mathrm{AlbB}, \mathrm{p}<0.0001$ ) and $28^{\circ} \mathrm{C}$ (unmanipulated vs. $w \mathrm{AlbB}, \mathrm{p}<0.0001$; Sua5B lysate vs. $w \mathrm{AlbB}, \mathrm{p}=0.002$; Table 2) relative to the controls and no significant effect of Wolbachia on malaria infection at $20^{\circ} \mathrm{C}$ (Table 2). However, when comparing the unadjusted means at $24^{\circ} \mathrm{C}$ to model estimates, we did not observe a significant difference in total sporozoite production in the $w \mathrm{AlbB}$ treatment group relative to the controls (Fig. 2d). This is most likely because the majority of the variance in the model is explained by the positive relationship between oocyst intensity and sporozoites produced per midgut. Thus, while the contribution of treatment in the model is small, it still significantly predicts the remaining variation unexplained by our covariate, with $w \mathrm{AlbB}$ infected mosquitoes producing fewer sporozoites (Supplementary Information Text S2). This result indicates that enhancement of oocyst intensity by $w \mathrm{AlbB}$ at $24^{\circ} \mathrm{C}$ was more or less counterbalanced by the negative effects $w \mathrm{AlbB}$ infection on parasite replication rate.

At $28^{\circ} \mathrm{C} w \mathrm{AlbB}$ caused significant reductions in overall infection intensity since both numbers of oocysts and sporozoite replication were negatively affected ( $S$ ua5 cells, $\mathrm{p}=0.003$; unmanipulated, $\mathrm{p}=$ 0.010 ; Table 2, Fig. 2d). We did see replicate effects at one temperature for both the sporozoite per oocyst and sporozoite per midgut analyses, likely due to variation in infection intensities between individual mice (Table 2). However, there were no significant interactions between replicate and temperature or treatment, so the replicate effects appear to have little influence on the results overall.

\section{Discussion}

Here we use a rodent malaria and somatically transinfected $A n$. stephensi as a model to investigate for the first time how changes in temperature influence the Wolbachia-malaria parasite interaction. We show that temperature significantly affects Wolbachia replication in the mosquito vector and alters the extent and apparent mode of action of transmission blocking. Temperature affected the replication kinetics of Wolbachia, establishment and replication of the malaria parasite, and the Wolbachia-parasite interaction. Infection with $w$ AlbB reduced oocyst intensity at $28^{\circ} \mathrm{C}$, increased oocyst intensity at $24^{\circ} \mathrm{C}$ and had no effect at $20^{\circ} \mathrm{C}$. Oocyst intensity is a common measure used to estimate parasite transmission blocking ${ }^{21,39}$. In this context, our results demonstrate that somatic infection with $w$ AlbB can partly block, enhance, or have no impact on infection depending on temperature. However, $w$ AlbB also appears to interfere with parasite replication, reducing the number of sporozoites produced per oocyst. When these effects are combined, $w$ AlbB reduces transmission potential strongly at $28^{\circ} \mathrm{C}$ but has no overall effect at $20^{\circ} \mathrm{C}$, or the thermal optimum for $P$. yoelii development, $24^{\circ} \mathrm{C}$. The potential for such marked temperature-dependence in transmission blocking phenotypes has not been considered previously.

Use of a rodent malaria complicates direct extension of our results to human malaria. Successful sporozoite invasion of the salivary glands by $P$. yoelii is inconsistent, especially at $28^{\circ} \mathrm{C}^{35}$, which is why we used sporozoite load within the midgut as our measure of overall infection intensity. Quantification of sporozoite intensity within the salivary glands, as is more reliable with P. falciparum, would provide a more definitive measure of pathogen blocking. We also used somatically infected mosquitoes rather than the recently developed stable transinfected line. While there has been little research to date comparing somatic infection and stable transinfection with Wolbachia, previous studies indicate that apart from the ovarian tissues, the density and distribution of Wolbachia within host tissues is similar between somatic and stable infections, both infection strategies impair development of $P$. falciparum at the oocyst stage $^{4,21}$, and modulate similar expression levels of immune genes $^{4,20,21,29,31}$. Further, the establishment and development rate of both human and rodent malarias are temperature sensitive, even though the absolute thermal performance profiles differ between species. Similar to earlier work investigating the effects of temperature on $P$. yoelii $i^{35}$, we found negative effects of temperature on $P$. yoelii oocyst establishment in the midgut at $28^{\circ} \mathrm{C}$. We would not expect $P$. falciparum oocyst establishment to be negatively affected until temperatures exceed $30^{\circ} \mathrm{C}^{40}$. However, this is not an unusual temperature for An. stephensi to experience in the field ${ }^{37}$. Moreover, $P$. vivax, which is the other key species of human malaria transmitted by $A n$. stephensi, has a lower temperature threshold than $P$. falciparum $^{37}$. Accordingly, there is little reason to believe the influence of temperature to be unique to our model system.

We found replication of $w \mathrm{AlbB}$ to be temperature sensitive, with an apparent optimum between $26^{\circ} \mathrm{C}$ and $28^{\circ} \mathrm{C}$. Studies in numerous systems show Wolbachia replication to increase towards some thermal optimum and then decline as temperatures increase further (Supplementary Information Table S1). Consistent with previous research conducted on An. gambiae, we found no effect of somatic infection on mosquito mortality, irrespective of whether or not a mosquito received a bloodmeal ${ }^{21}$. At $28^{\circ} \mathrm{C}$, we found a reduction in oocyst prevalence and intensity due to $w \mathrm{AlbB}$. These results are similar to those reported for $P$. falciparum in the stably transinfected line of An. stephensi at $27^{\circ} \mathrm{C}^{4}$. At cooler temperatures we found no effects on prevalence but an increase in oocyst intensity (significant at $24^{\circ} \mathrm{C}$ ). This is in accord with studies on another rodent malaria, $P$. berghei, which showed no impact on prevalence, but enhancement of oocyst intensities in somatically transinfected An. gambiae housed at $19^{\circ} \mathrm{C}^{39}$.

The mechanisms underpinning the diverse transmission blocking phenotypes require further study. There is some evidence that $w \mathrm{AlbB}$ induces the production of reactive oxygen species (ROS), as well as the upregulation of immune genes like TEP1 and $L I R M-1^{20}$, and that this can inhibit initial Plasmodium infection ${ }^{4,21}$. How this would lead to enhancement under certain temperatures is unclear but the timing of midgut microbiota proliferation, ROS production, immune gene expression, and parasite kinetics directly following the blood meal might be crucial for parasite blocking at early stages of malaria infection. These different processes are all likely temperature sensitive but need not show identical responses ${ }^{34}$. In addition, the rodent malarias might initiate different responses since ookinetes typically form and migrate through the midgut more rapidly (12-24 hr post-infection) than $P$. falciparum $(48 \mathrm{hr})^{41}$. Other elements of mosquito immune response also differ between rodent and human malaria. The Toll pathway primarily regulates mosquito defense at the oocyst stage against rodent malarias, while the IMD pathway is important for defense against $P$. falciparum $^{42-44}$. Such differences might lead to contrasting patterns of blocking depending on which genes are modulated by $w$ AlbB infection and how immune mechanisms are affected by temperature ${ }^{21}$. Variation in $w$ AlbB densities within the mosquito could also play a role, although previous research on somatically infected An. gambiae suggests there is no direct relationship between oocyst burdens and $w$ AlbB densities for either P. berghei or P. falciparum ${ }^{21,39}$.

The impact of $w$ AlbB infection on sporozoite development is a completely novel finding. Overall, $w$ AlbB reduced the number of sporozoites produced per oocyst across experimental temperatures. This effect counterbalanced the substantial enhancement of oocysts at $24^{\circ} \mathrm{C}$ leading to no net impact of $w \mathrm{AlbB}$ on overall infection intensity, while further strengthening blocking at $28^{\circ} \mathrm{C}$. The link with oocyst density suggests that the effect could be due in part to direct or indirect resource-mediated competition with sporozoite replication inhibited when $w$ AlbB and/or malaria parasite density are high ${ }^{45}$. The presence of Wolbachia, combined with host resource demands, could limit the amount or accessibility of resources available for Plasmodium development ${ }^{15}$. Wolbachia and Plasmodium are amino acid heterotrophs and have important and potentially overlapping lipid requirements ${ }^{46-49}$. Whether Wolbachia infection further affects the viability and invasibility of Plasmodium sporozoites needs to be further investigated in this system. 
In this study we demonstrate complex effects of ambient temperature on the Wolbachia-malaria interaction that alter the nature and extent of pathogen blocking. Mean temperatures and daily temperature ranges in natural transmission environments can far exceed those considered here. Based on our data it would be remarkable if the interactions between Wolbachia, the mosquito vector and human malaria parasites were unaffected by such variation. Moreover, the impact of $w$ AlbB on species such as P. vivax or on mixed infections remains unexplored; the potential for enhancement need not be limited to rodent malaria. Further development of this promising control technology requires an improved understanding of how mosquitoes, Wolbachia and malaria parasites will interact in diverse transmission settings.

\section{Methods}

Mosquito rearing, experimental design, and infections. We reared Anopheles stephensi (Liston) under standard insectary conditions at $26 \pm 0.5^{\circ} \mathrm{C}, 80 \%$ humidity, and a 12 h light: $12 \mathrm{~h}$ dark photo-period and on a $10 \%$ glucose solution $\operatorname{diet}^{32}$. Wolbachia (wAlbB, isolated from in vitro cultivation of Wolbachia pipientis in an Ae. albopictus cell line $\mathrm{e}^{50}$ ) was cultured and extracted from $w$ AlbB-infected Sua5B Anopheles cells and suspended in Schneider's insect cell media as previously described ${ }^{31,51,52}$. On day three post-emergence, adult female mosquitoes were randomly allocated to one of three treatment groups: 1) unmanipulated, 2) injected with $0.2 \mu \mathrm{L}$ Sua5B cell lysates and Schneider's insect cell media, or 3) injected with $0.2 \mu \mathrm{L}$ of Wolbachia $\left(5 \times 10^{6}\right.$ Wolbachia per $\mathrm{mL} ; 1,000$ bacteria per dose $)$. Mosquitoes that were challenged with Sua5B cell lysates or Wolbachia received intrathoracic injection into the anepisternal cleft $^{32}$ with a mouth pipette and microcapillary glass needle. Immediately after injection, 1200 mosquitoes from each treatment group ( 3600 mosquitoes total) were placed into cages $(20 \times 20 \times 20 \mathrm{~cm}, 80$ mosquitoes per cage) and randomly distributed across five Percival incubators set to different experimental temperatures $\left(20^{\circ} \mathrm{C}, 22^{\circ} \mathrm{C}, 24^{\circ} \mathrm{C}, 26^{\circ} \mathrm{C}\right.$, and $28^{\circ} \mathrm{C} \pm 0.5^{\circ} \mathrm{C}$, $80 \%$ humidity at a $12 \mathrm{hr}$ light: $12 \mathrm{hr}$ dark photo-period) and three replicates. These experimental temperatures reflect a realistic temperature range for $P$. yoelii transmission, which has a thermal optimum around $24^{\circ} \mathrm{C}$ for parasite transmission and development ${ }^{35,53}$

To maintain experimental tractability, on day eight post-infection with Wolbachia, mosquitoes from each treatment group and replicate that were housed across three experimental temperatures $\left(20^{\circ} \mathrm{C}, 24^{\circ} \mathrm{C}\right.$, and $\left.28^{\circ} \mathrm{C}\right)$ received a malaria infectious bloodmeal from a mouse (C57 BI/6, >6 weeks old) infected with $10^{5}$ Plasmodium yoelii parasites (clone 17XNL, from the WHO Registry of Standard Malaria Parasites, University of Edinburgh, UK) four days prior. Individuals that did not feed were removed from the cage. Mosquitoes were maintained at $24^{\circ} \mathrm{C}$, the thermal optimum for Plasmodium yoelii development and replication ${ }^{35}$, for two hours following the $30 \mathrm{~min}$ bloodfeed to allow for gamete and zygote formation. After infection, individuals were placed back into their respective temperature treatments and provided with cotton balls moistened with $10 \%$ glucose offered ad libitum. Throughout the duration of the experiment, we counted and removed dead mosquitoes in each cage daily to quantify the effects of treatment and temperature on daily mosquito mortality.

\section{Quantifying the effects of temperature on Wolbachia dissemination. We} destructively sampled 10 mosquitoes per cage across all temperatures on days 8,12 , 16 , and 20 post-Wolbachia infection. Mosquitoes were killed and immediately frozen at $-80^{\circ} \mathrm{C}$ for future qPCR analyses. DNA was extracted from whole mosquito carcasses using the insect supplement in the 96-well DNeasy Blood and Tissue kit (Qiagen, Inc.) as per the manufacturer's protocol. We performed all qPCR analyses on an ABI Prism 7500 Sequence Detection System (TaqMan) using the Rotor-Gene SYBR Green PCR kit as per the manufacturer's protocol (Qiagen, Inc.) and the following cycler conditions ( 40 cycles total: activation, $95^{\circ} \mathrm{C}$ for $5 \mathrm{~min}$; denaturation, $40 \mathrm{cycles}$ run at $95^{\circ} \mathrm{C}$ for $5 \mathrm{sec}$ each; and annealing and extension, $60^{\circ} \mathrm{C}$ for $30 \mathrm{sec}$ ). $w$ AlbB was amplified from mosquito carcasses with modified GF and BR primers which specifically bind to the $w s p$ gene $e^{54}$. The relative abundance of $w$ AlbB strain was determined after normalization to the mosquito single-copy $r p S 7$ gene ${ }^{21,32}$. All qPCRs were completed in triplicate, and the average efficiency of each assay was determined by quantifying the slope of a standard curve of five $1: 10$ serial dilutions from a positive sample in duplicate. Wolbachia densities (presented as a fold-change relative to $r p S 7$ DNA) were generated using the qGENE software ${ }^{55}$.

Quantifying the effects of temperature on Plasmodium blocking. We compared how oocyst prevalence, intensity, and sporozoite replication varied with Wolbachia infection and temperature $\left(20^{\circ} \mathrm{C}, 24^{\circ} \mathrm{C}\right.$, and $\left.28^{\circ} \mathrm{C}\right)$. To quantify parasite prevalence and intensity, we removed and immediately killed 20 mosquitoes from each treatment and temperature group with chloroform and dissected their midguts in $1 \times$ phosphate-buffered saline solution under a standard dissecting scope. Using a compound microscope, we noted whether a midgut was infected or uninfected, and counted the number of Plasmodium oocysts that had established in each infected midgut. Midguts were then immediately stored in 95\% ethanol for future molecular analyses to assess Plasmodium replication and potential sporozoite production. To account for thermal shifts in Plasmodium development rates ${ }^{35}$, dissections were staggered to ensure midguts were sampled at approximately the same stage of oocyst development (day 7,8 , and 10 post-malaria infection for $24^{\circ} \mathrm{C}, 28^{\circ} \mathrm{C}$, and $20^{\circ} \mathrm{C}$, respectively).

Due to inconsistent salivary gland invasion by $P$. yoelii sporozoites, we used sporozoite production per midgut and per oocyst as proxies to estimate transmission potential. To assess sporozoite production, DNA was extracted from individual midguts collected during oocyst dissection using the E.Z.N.A. MicroElute Genomic DNA kit (Omega Bio-Tek, as per the manufacturer's protocol). DNA was eluted in $20 \mu \mathrm{L}$ of elution buffer, and the number of parasite genomes present in midguts was quantified using a previously developed qPCR assay ${ }^{56}$. Sporozoite production per oocyst was evaluated by dividing the total number of sporozoites per midgut by the number of oocysts quantified for each midgut.

Statistical analyses. All statistical analyses for these experiments were run in IBM SPSS Statistics 21.0 (IBM Corporation). Full factorial models from generalized linear model (GZLM) analysis were reduced through backward elimination of nonsignificant interactions. We assessed goodness of fit of the final models through model deviance, log likelihood values, and model residuals. Covariates included in GZLMs were centred on their grand mean, and adjusted Bonferroni post-hoc tests were used to identify significant pair-wise comparisons. For all dependent variables analyzed, we included the following factors in our model analysis: temperature (Wolbachia densities and mosquito survival, $20^{\circ} \mathrm{C}, 22^{\circ} \mathrm{C}, 24^{\circ} \mathrm{C}, 26^{\circ} \mathrm{C}$, and $28^{\circ} \mathrm{C}$; Plasmodium blocking, $20^{\circ} \mathrm{C}, 24^{\circ} \mathrm{C}$, and $28^{\circ} \mathrm{C}$ ), treatment (unmanipulated, Sua5B, and $w$ AlbB), and replicate.

We used a GZLM assuming a normal distribution to determine how Wolbachia density was affected by our experimental treatment groups. In addition to the fixed factors mentioned above, we included sampling date post-Wolbachia infection in our model analysis (day 8, 12, 16, and 20 PI). We used a Poisson GZLM to quantify the effects of experimental treatment on the average number of infected mosquitoes and a negative binomial (log link function) GZLM to quantify the effects of experimental treatment on oocyst intensity. Because of differences in parasite replication rates due to temperature, we ran a series of GZLM analyses (assuming either a normal distribution on transformed sporozoite data or a gamma distribution on untransformed data) on the total number of Plasmodium sporozoites per midgut and per oocyst independently for each temperature. Because midguts with more oocysts likely produce more sporozoites, we also included oocyst intensity as a covariate in both analyses. Finally, we used Kaplan-Meier survival analyses to generate cumulative daily survival estimates to determine the effects of temperature and treatment on daily mosquito survival throughout the course of the experiment.

1. Organization, W. H. World Malaria Report. 195 (2012).

2. Dong, Y. et al. Engineered Anopheles immunity to Plasmodium infection. PLoS Pathog. 7, 1-12 (2011).

3. Isaacs, A. T. et al. Engineered resistance to Plasmodium falciparum development in transgenic Anopheles stephensi. PLoS Pathog. 7, 1-12, doi:e100201710.1371/ journal.ppat.1002017 (2011).

4. Bian, G. et al. Wolbachia invades Anopheles stephensi populations and induces refractoriness to Plasmodium infection. Science 340, 748-751, doi:10.1126/ science.1236192 (2013)

5. Yoshida, S. et al. Hemolytic C-type lectin CEL-III from sea cucumber expressed in transgenic mosquitoes impairs malaria parasite development. PLoS Pathog. 3 , 1962-1970, doi:e19210.1371/journal.ppat.0030192 (2007).

6. Li, C. Y., Marrelli, M. T., Yan, G. Y. \& Jacobs-Lorena, M. Fitness of transgenic Anopheles stephensi mosquitoes expressing the SM1 peptide under the control of a vitellogenin promoter. J. Hered. 99, 275-282, doi:10.1093/jhered/esn004 (2008).

7. Cook, P. E. \& McGraw, E. A. Wolbachia pipientis: an expanding bag of tricks to explore for disease control. Trends Parasitol. 26, 373-375, doi:10.1016/ j.pt.2010.05.006 (2010).

8. Iturbe-Ormaetxe, I., Walker, T. \& Neill, S. L. O. Wolbachia and the biological control of mosquito-borne disease. EMBO Rep. 12, 508-518, doi:10.1038/ embor.2011.84 (2011).

9. McGraw, E. A. \& O’Neill, S. L. Beyond insecticides: new thinking on an ancien problem. Nat. Rev. Microbiol. 11, 181-193, doi:10.1038/nrmicro2968 (2013).

10. Ricci, I. et al. Symbiotic control of mosquito borne disease. Pathog. Gl. Health 106, 380-385, doi:10.1179/2047773212y.0000000051 (2012).

11. Hughes, G. L. \& Rasgon, J. L. Transinfection: a method to investigate Wolbachiahost interactions and control arthropod-borne disease. Insect Mol. Biol. n/a-n/a, doi:10.1111/imb.12066 (2014)

12. Bourtzis, K. et al. Harnessing mosquito-Wolbachia symbiosis for vector and disease control. Acta Trop., doi:http://dx.doi.org/10.1016/j.actatropica.2013.11. 004 (in press)

13. Glaser, R. L. \& Meola, M. A. The native Wolbachia endosymbionts of Drosophila melanogaster and Culex quinquefasciatus increase host resistance to West Nile virus infection. PLoS ONE 5, 1-11, doi:e1197710.1371/journal.pone.0011977 (2010).

14. Hussain, M. et al. Effect of Wolbachia on replication of West Nile virus in a mosquito cell line and adult mosquitoes. J. Virol. 87, 851-858, doi:10.1128/ jvi.01837-12 (2013) 
15. Moreira, L. A. et al. A Wolbachia symbiont in Aedes aegypti limits infection with dengue, chikungunya, and Plasmodium. Cell 139, 1268-1278, doi:10.1016/ j.cell.2009.11.042 (2009).

16. Bian, G., Xu, Y., Lu, P., Xie, Y. \& Xi, Z. The endosymbiotic bacterium Wolbachia induces resistance to dengue virus in Aedes aegypti. PLoS Pathog. 6, 1-12, doi:e100083310.1371/journal.ppat.1000833 (2010).

17. Blagrove, M. S. C., Arias-Goeta, C., Di Genua, C., Failloux, A.-B. \& Sinkins, S. P. A Wolbachia wMel transinfection in Aedes albopictus is not detrimental to host fitness and inhibits Chikungunya virus. PLoS Negl. Trop. Dis. 7, 1-7, doi:e215210.1371/journal.pntd.0002152 (2013).

18. van den Hurk, A. F. et al. Impact of Wolbachia on infection with Chikungunya and yellow fever viruses in the mosquito vector Aedes aegypti. PLoS Negl. Trop. Dis. 6, 1-9, doi:e189210.1371/journal.pntd.0001892 (2012).

19. Kambris, Z., Cook, P. E., Phuc, H. K. \& Sinkins, S. P. Immune activation by lifeshortening Wolbachia and reduced filarial competence in mosquitoes. Science 326, 134-136, doi:10.1126/science.1177531 (2009).

20. Kambris, Z. et al. Wolbachia stimulates immune gene expression and inhibits Plasmodium development in Anopheles gambiae. PLoS Pathog. 6, 1-9, doi:e100114310.1371/journal.ppat.1001143 (2010).

21. Hughes, G. L., Koga, R., Xue, P., Fukatsu, T. \& Rasgon, J. L. Wolbachia infections are virulent and inhibit the human malaria parasite Plasmodium falciparum in Anopheles gambiae. PLoS Pathog. 7, 1-8, doi:e100204310.1371/ journal.ppat.1002043 (2011).

22. McMeniman, C. J. et al. Stable introduction of a life-shortening Wolbachia infection into the mosquito Aedes aegypti. Science 323, 141-144, doi:10.1126/ science.1165326 (2009)

23. Suh, E., Mercer, D. R., Fu, Y. \& Dobson, S. L. Pathogenicity of life-shortening Wolbachia in Aedes albopictus after transfer from Drosophila melanogaster. Appl. Environ. Microbiol. 75, 7783-7788, doi:10.1128/aem.01331-09 (2009).

24. Calvitti, M., Moretti, R., Lampazzi, E., Bellini, R. \& Dobson, S. L. Characterization of a new Aedes albopictus (Diptera: Culicidae)-Wolbachia pipientis (Rickettsiales: Rickettsiaceae) symbiotic association generated by artificial transfer of the wPip strain from Culex pipiens (Diptera: Culicidae). J. Med. Entomol. 47, 179-187, doi:10.1603/me09140 (2010).

25. Moreira, L. A. et al. Human probing behavior of Aedes aegypti when infected with a life-shortening strain of Wolbachia. PLoS Negl. Trop. Dis. 3, 1-6, doi:e56810.1371/journal.pntd.0000568 (2009).

26. Turley, A. P., Moreira, L. A., O’Neill, S. L. \& McGraw, E. A. Wolbachia infection reduces blood-feeding success in the dengue fever mosquito, Aedes aegypti. PLoS Negl. Trop. Dis. 3, 1-6, doi:e51610.1371/journal.pntd.0000516 (2009).

27. Werren, J. H. Biology of Wolbachia. Annu. Rev. Entomol. 42, 587-609, doi:10.1146/annurev.ento.42.1.587 (1997).

28. Hoffmann, A. A. et al. Successful establishment of Wolbachia in Aedes populations to suppress dengue transmission. Nature 476, 454-U107, doi:10.1038/nature10356 (2011).

29. Pan, X. et al. Wolbachia induces reactive oxygen species (ROS)-dependent activation of the Toll pathway to control dengue virus in the mosquito Aedes aegypti. Proc. Natl. Acad. Sci. U. S. A. 109, E23-E31, doi:10.1073/ pnas.1116932108 (2012)

30. Hussain, M., Frentiu, F. D., Moreira, L. A., O’Neill, S. L. \& Asgari, S. Wolbachia uses host microRNAs to manipulate host gene expression and facilitate colonization of the dengue vector Aedes aegypti. Proc. Natl. Acad. Sci. U. S. A. 108, 9250-9255, doi:10.1073/pnas.1105469108 (2011).

31. Hughes, G. L. et al. Wolbachia infections in Anopheles gambiae cells transcriptomic characterization of a novel host-symbiont interaction. PLoS Pathog. 7, 1-11, doi:e100129610.1371/journal.ppat.1001296 (2011).

32. Murdock, C. C. et al. Complex effects of temperature on mosquito immune function. P. Roy. Soc. Lond. B. Bio. 279, 3357-3366, doi:10.1098/rspb.2012.0638 (2012).

33. Murdock, C. C., Moller-Jacobs, L. L. \& Thomas, M. B. Complex environmental drivers of mosquito resistance: a challenge for reductionist immunology. P. Roy. Soc. Lond. B. Bio. 280, 2013-2030, doi:10.1098/rspb.2013.2030 (2013).

34. Murdock, C. C., Paaijmans, K. P., Read, A. F., Cox-Foster, D. \& Thomas, M. B. Rethinking vector immunology: the role of environmental temperature in shaping resistance. Nat. Rev. Microbiol. 10, 869-876 (2012).

35. Paaijmans, K. P., Blanford, S., Chan, B. H. K. \& Thomas, M. B. Warmer temperatures reduce the vectorial capacity of malaria mosquitoes. Biol. Lett. 8, 465-468, doi:10.1098/rsbl.2011.1075 (2012).

36. Paaijmans, K. P. et al. Influence of climate on malaria transmission depends on daily temperature variation. Proc. Natl. Acad. Sci. U. S. A. 107, 15135-15139, doi:10.1073/pnas.1006422107 (2010)

37. Cator, L. J. et al. Characterizing microclimate in urban malaria transmission settings: a case study from Chennai, India. Malar. J. 12, 1-10, doi:8410.1186/ $1475-2875-12-84$ (2013).

38. Blanford, J. I. et al. Implications of temperature variation formalaria parasite development across Africa. Sci. Rep 3, 1300, DOI:10.1038/srep01300 (2013).

39. Hughes, G. L., Vega-Rodriguez, J., Xue, P. \& Rasgon, J. L. Wolbachia strain wAlbB enhances infection by the rodent malaria parasite Plasmodium berghei in Anopheles gambiae mosquitoes. Appl. Environ. Microbiol. 78, 1491-1495, doi:10.1128/aem.06751-11 (2012).
40. Mordecai, E. A. et al. Optimal temperature for malaria transmission is dramatically lower than previously predicted. Ecol. Lett. 16, 22-30, doi:10.1111/ ele.12015 (2013)

41. Vaughan, J. A. Population dynamics of Plasmodium sporogony. Trends Parasitol. 23, 63-70, doi:10.1016/j.pt.2006.12.009 (2007)

42. Dong, Y. M. et al. Anopheles gambiae immune responses to human and rodent Plasmodium parasite species. PLoS Pathog. 2, 513-525, doi:e52 10.1371/ journal.ppat.0020052 (2006).

43. Garver, L. S., Dong, Y. M. \& Dimopoulos, G. Casper controls resistance to Plasmodium falciparum in diverse Anopheline species. PLoS Pathog. 5, 1-12, doi:e100033510.1371/journal.ppat.1000335 (2009).

44. Garver, L. S. et al. Anopheles Imd pathway factors and effectors in infection intensity-dependent anti-Plasmodium action. PLoS Pathog. 8, 1-12, doi:e100273710.1371/journal.ppat.1002737 (2012).

45. Pollitt, L. C. et al. Costs of crowding for the transmission of malaria parasites. Evol. Appl. 6, 617-629, doi:10.1111/eva.12048 (2013).

46. Wu, M. et al. Phylogenomics of the reproductive parasite Wolbachia pipientis wMel: a streamlined genome overrun by mobile genetic elements. PLoS Biol. 2, 327-341, doi:e6910.1371/journal.pbio.0020069 (2004).

47. Atella, G. C., Bittencourt-Cunha, P. R., Nunes, R. D., Shahabuddin, M. \& SilvaNeto, M. A. C. The major insect lipoprotein is a lipid source to mosquito stages of malaria parasite. Acta Trop. 109, 159-162, doi:10.1016/j.actatropica.2008.10.004 (2009).

48. Gardner, M. J. et al. Genome sequence of the human malaria parasite Plasmodium falciparum. Nature 419, 498-511, doi:10.1038/nature01097 (2002).

49. Caragata, E. P. et al. Dietary cholesterol modulates pathogen blocking by Wolbachia. PLoS Pathog. 9, e1003459, doi:10.1371/journal.ppat.1003459 (2013).

50. O'Neill, S. L. et al. In vitro cultivation of Wolbachia pipientis in an Aedes albopictus cell line. Insect Mol. Biol. 6, 33-39, doi:10.1046/j.1365-2583.1997.00157.x (1997).

51. Rasgon, J. L., Gamston, C. E. \& Ren, X. Survival of Wolbachia pipientis in cell-free medium. Appl. Environ. Microbiol. 72, 6934-6937, doi:10.1128/aem.01673-06 (2006).

52. Rasgon, J. L., Ren, X. \& Petridis, M. Can Anopheles gambiae be infected with Wolbachia pipientis? Insights from an in vitro system. Appl. Environ. Microbiol. 72, 7718-7722, doi:10.1128/aem.01578-06 (2006).

53. Sato, Y., Matsuoka, H., Araki, M., Ando, K. \& Chinzei, Y. Effect of temperature to Plasmodium berghei and P. yoelii on mosquito stage in Anopheles stephensi.Jpn. J. Parasitol. 45, 98-104 (1996).

54. Hughes, G. L., Pike, A. D., Xue, P. \& Rasgon, J. L. Invasion of Wolbachia into Anopheles and other insect germlines in an ex vivo organ culture system. PLoS ONE 7, 1-8, doi:e36277 10.1371/journal.pone.0036277 (2012).

55. Joehanes, R. \& Nelson, J. C. QGene 4.0, an extensible Java QTL-analysis platform. Bioinformatics 24, 2788-2789, doi:10.1093/bioinformatics/btn523 (2008).

56. Bell, A. S., De Roode, J. C., Sim, D. \& Read, A. F. Within-host competition in genetically diverse malaria infections: parasite virulence and competitive success Evolution 60, 1358-1371, doi:10.1554/05-611.1 (2006)

\section{Acknowledgments}

We thank members of the Thomas and Read lab groups for discussion and J. Teeple for insectary support and R. Schneider for assistance with Wolbachia culture and purification. This research was funded by the following grants: NSF-NIH EID (EF-0914384), NIH-R21 (AI096036-01), NIH-R21 (AI070178), and NIH-R21 (AI088311). The content of this paper is solely the responsibility of the authors and does not necessarily represent the official views of the National Institute of Allergy and Infectious Diseases, and the National Institutes of Health. This project was also assisted by a grant from the Pennsylvania Department of Health using Tobacco Settlement Funds. The Department specifically disclaims responsibility for any analyses, interpretations or conclusions.

\section{Author contributions}

C.C.M. contributed to conceiving and designing experiments, running the experiments, analyzing data associated with the experiments, and in writing the manuscript. S.B. contributed to designing and running the experiments. G.L.H. contributed to designing the experiment, provided tools and techniques for the experiment, and in writing the manuscript. J.L.R. contributed by providing resources for the experiment and in writing the manuscript. M.B.T. contributed to conceiving the experiments, providing resources for the experiments, and in writing the manuscript.

\section{Additional information}

Supplementary information accompanies this paper at http://www.nature.com/ scientificreports

Competing financial interests: The authors declare no competing financial interests. How to cite this article: Murdock, C.C. et al. Temperature alters Plasmodium blocking by Wolbachia. Sci. Rep. 4, 3932; DOI:10.1038/srep03932 (2014)

This work is licensed under a Creative Commons Attribution 3.0 Unported license. To view a copy of this license, visit http://creativecommons.org/licenses/by/3.0 